\title{
Removal of Hazardous Brilliant Cresyl Blue Dye Utilizing Aluminum Oxide as Photocatalyst
}

\author{
Hazim Yahya Al-Gubury ${ }^{1, *}$, Hajir S. Alteemi ${ }^{1}$, Ali Malik Saad ${ }^{2}$, and Rafal R. Al-Shamary ${ }^{1}$ \\ ${ }^{1}$ Department of Chemistry, College of Science for Women, University of Babylon, Iraq \\ ${ }^{2}$ Department of Biology, College of Science for Women, University of Babylon, Iraq
}

* Corresponding author:

email: h.yahya40@yahoo.com

Received: November 8, 2017

Accepted: March 15, 2018

DOI: $10.22146 /$ ijc.30135

\begin{abstract}
Photocatalytic degradation of brilliant cresyl blue stain has been done by irradiating the solution pigment with a solar light in the presence of aluminum oxide. The effect of important reaction has included many parameters such as catalyst mass, the initial concentration of brilliant cresyl blue dye, the effect of temperature, and initial $P$ has been investigated in a batch reactor. All experiments are determined by usage of UVvisible spectrophotometer analyzer. The optimal concentration of catalytic has observed at $0.17 \mathrm{~g} / 100 \mathrm{~cm}^{3}$ while the typical concentration of stain was recorded at $50 \mathrm{mg} / \mathrm{cm}^{3}$. Photocatalytic break down of shining cresyl blue pigment was favorable in the $P h 10$ and $8.44 \mathrm{~mW} / \mathrm{cm}^{2}$ light intensity. This type of degradation of the present dye study has obeyed the pseudo-first-order reaction. Photocatalytic degradation of brilliant cresyl blue dye has studied by using various concentrations of aluminum oxide. This concentration was suspended in an aqueous solution of dye which has irradiated by solar radiation in a vessel reactor at room temperature with $10 \mathrm{~cm}^{3} / \mathrm{min}$ air bubble was passed through the solution.
\end{abstract}

Keywords: photocatalytic; degradation; brilliant cresyl blue; aluminum oxide

\section{- INTRODUCTION}

In the last years, neutral and synthetic stains have huge applied in textile industry for staining of cotton, silk, and wood. These kinds of pigments have many undesirable properties such as color, taste, and toxicity. These unwanted features lead to environmental contamination of the surface water as well as groundwater by releasing the toxic and colored effluents which affected on the human health, therefore should be treated and discharged into the drinking water [1-3]. Development oxidation processes (AOPs) are one of the most appealing techniques for the enhanced oxidation of toxic contaminants. It has the ability to transform the pollutants into harmless materials. Metal oxides are widely used as the active phases in heterogeneous catalysis for treatment of wastewater, and other applications [4]. In the past few years catalyst have drawn much attention to the breakdown of organic pollutants such as pigments, pesticides, and volatile organic compounds by usage ultraviolet light irradiation [5]. Basically, this method has started with the generation of strong oxidizing agents such as hydroxyl radicals $\left({ }^{\circ} \mathrm{OH}\right)(\mathrm{E}=2.8 \mathrm{~V})$. This reactive radical capable of mineralizing organic contaminants [6]. When aluminum oxide [7] has irradiated by utilizing UV light with energy equal to or greater than the bandgap. In this process, the electrons are promoted from the valence band to the conduction band of the aluminum oxide semiconducting oxide to give electron-hole pairs. The valence bundle hole $\left(\mathrm{h}^{+}\right)$ potential is positive enough to generate hydroxyl radicals at the surface as well as the conduction band electron $\left(\mathbf{e}^{-}\right)$is negative adequate to reduce the oxygen molecules have found in the solution, which in turn leads to the generation of another series of hydroxyl radicals [8-9]. Many semiconductors such as $\mathrm{ZnO}, \mathrm{TiO}_{2}$, and CdS have used to destroy organic and inorganic contaminants instance manufacture stains, insects, heavy metals in sewage by employment photocatalytic 
<smiles></smiles>

Fig 1. Brilliant cresyl blue stain structure

destruction process. Brilliant cresyl blue is a supravital stain has used for counting reticulocytes. It has classified as an oxazine pigment. The chemical formula of this stain is $\left(\mathrm{C}_{17} \mathrm{H}_{20} \mathrm{ClN}_{3} \mathrm{O}\right)_{2} \cdot \mathrm{ZnCl}_{2}$, molar mass $(385.9 \mathrm{~g} / \mathrm{mol})$. A basic oxazine dye is using for staining the reticulum in young erythrocytes (reticulocytes). This sort of cells has commonly utilized for studies of multifarious biological systems [10].

\section{- EXPERIMENTAL SECTION}

\section{Materials}

The chemicals have used in this work are listed below, and all chemicals are standard without further purification, aluminum oxide $\left(\mathrm{Al}_{2} \mathrm{O}_{3}, 98 \%\right.$ purity, Merck); Brilliant cresyl blue (Sigma-Aldrich), sodium hydroxide $(\mathrm{NaOH}$, Fluka AG), hydrochloric acid ( $\mathrm{HCl}$, Fluka $\mathrm{AG})$.

\section{Instrumentation}

UV spectrophotometer has utilized to monitor the photocatalytic reaction.

\section{Procedure}

\section{Photocatalytic degradation of brilliant cresyl blue}

The photocatalytic breakdown experiments of the liquid solution of brilliant cresyl blue stain. In this experience, the pigment was exposed to teach solar energy and aluminum oxide in a batch reactor. This reactor consists of the cylindrical annular-type reactor has contained two parts. The first part was an outside thimble, running water was passed through the thimble to cool the reaction solution. Owing to the continued cooling, the temperature of the reaction solution was maintained at room temperature. The second part was an inside thimble with the reaction solution $\left(100 \mathrm{~cm}^{3}\right)$ was put in the reaction chamber. The photocatalytic breakdown of dye was conducted under solar radiation. All experiments of photocatalytic degradation processes of stain have performed by mixing $0.17 \mathrm{~g} / 100 \mathrm{~cm}^{3}$ of the catalyst with $50 \mathrm{ppm}$ of the dye solution. In order to confirm the adsorption equilibrium between the surface of the catalyst and pigment. Firstly, the suspension solution of aluminum oxide and the brilliant cresyl blue dye solution has been stirred in the dark for $30 \mathrm{~min}$ to allow the dark adsorption to attain balance. Then, the solution of dye has bubbled with air $\left(10 \mathrm{~cm}^{3} / \mathrm{min}\right)$ during the irradiation. After that, $2 \mathrm{~cm}^{3}$ of the suspension reaction mixture was withdrawn every $10 \mathrm{~min}$. Later, centrifugation has done at $4000 \mathrm{rpm}$ to remove any residual antimony trioxide particles. Finally, all samples have taken to analyzed at maximum absorption band by $\mathrm{UV}$-Vis spectrophotometer.

\section{- RESULTS AND DISCUSSION}

\section{The Impact of Aluminum Oxide Mass on Photocatalytic Degradation of Brilliant Cresyl Blue}

The effect of catalyst loading on the rates of destruction of the bright cresyl blue stain as shown in Fig. 2. From Fig. 2 the rate of destroying of brilliant cresyl blue dye has increased with an increase in catalyst weight from 0.07 to $0.17 \mathrm{~g} / 100 \mathrm{~cm}^{3}$. Additional, increase in catalyst amount has resulted in a decrease in the rate photocatalytic degradation process of shining cresyl blue pigment. The maximum percentage of photodegradation was obtained at $0.17 \mathrm{~g} / 100 \mathrm{~cm}^{3}$ of aluminum oxide, which can provide the highest absorption of light. Moreover, increasing the number of active sites with increasing the masses of aluminum oxide leads to enhance the number of adsorbed brilliant cresyl blue stain molecules, consequently increase the percentage of photodegradation of dye. The average has decreased at catalyst loadings beyond the optimum value is $0.17 \mathrm{~g} / 100 \mathrm{~cm}^{3}$. This optimal value has recorded by the stain. This can be attributed to the penetration of light during the dense catalyst suspension becomes difficult. At higher catalyst loading, the catalyst particles also scatter the incident photons, and hence the availability of the photon flux to the solution has decreased. This decrease was accompanied by a decrease in the photocatalytic breakdown rates [11]. 


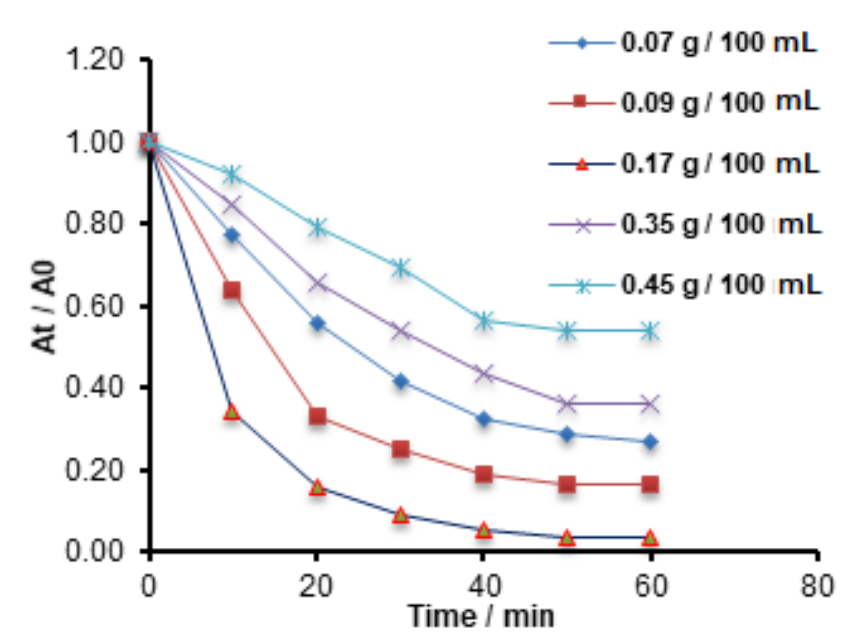

Fig 2. Effect of photocatalyst loading on photodegradation of brilliant cresyl blue stain by usage solar radiation, initial condition: $50 \mathrm{ppm}$ brilliant cresyl blue pigment and $\mathrm{pH}=10$

\section{The Impacting of Initial Brilliant Cresyl Blue Concentration on Photocatalytic Degradation Processes}

Fig. 3 has revealed the average decreases of photocatalytic degradation with the increasing of initial brilliant cresyl blue dye concentration. This behavior could be explained: the concentration $50 \mathrm{ppm}$ was the optimum concentration to cover the largest area of the aluminum oxide particles. The maximum absorption of exciting photons leads to generate a higher concentration of the activated aluminum oxide semiconductor. At a high brilliant cresyl blue dye, the concentration beyond 50 ppm, the presumption is that the active sites are covered by brilliant cresyl blue dye that can cause reduced generation of an electron-hole pair. Subsequently, this reduction in production of an electron-hole pair leads up to drooping of photodegradation efficiency. Another reason which may be responsible for the reduction in photocatalytic degradation efficiency is the excess of brilliant cresyl blue pigment. This excessive in stain leads to prevent the penetration of light meantime the successive layers of radiant cresyl blue dye on the aluminum oxide surface [12-13].

Plotting $\ln \mathrm{A}_{0} / \mathrm{A}_{\mathrm{t}}$ versus Time has given straight line as shown in Fig. 4. These results have shown that the photocatalytic destruction of shiny cresyl blue tincture

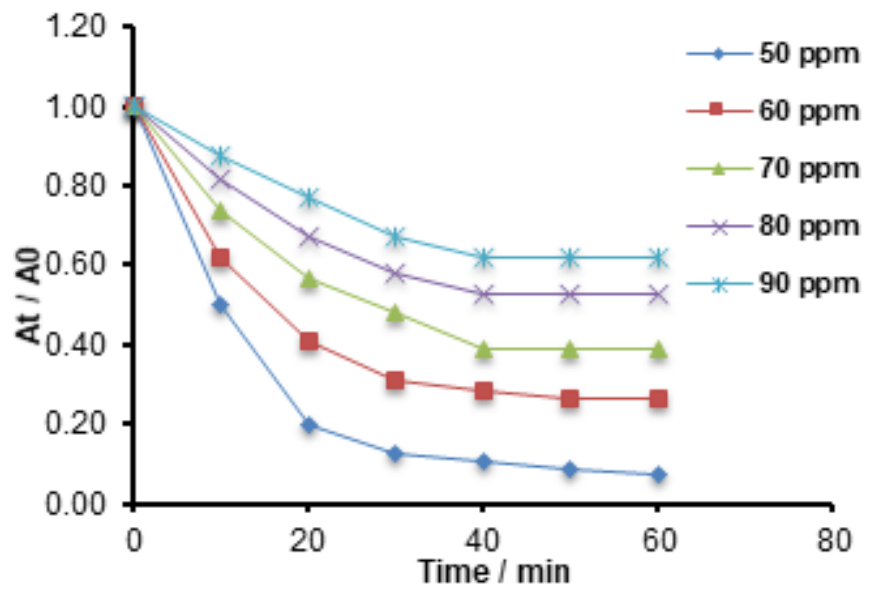

Fig 3. The change of $\left(A / A_{0}\right)$ with irradiation time at a concentration of brilliant cresyl blue by utilizing solar radiation, initial condition: $0.17 \mathrm{~g} / 100 \mathrm{~cm}^{3}$ aluminum oxide and $\mathrm{pH}=10$

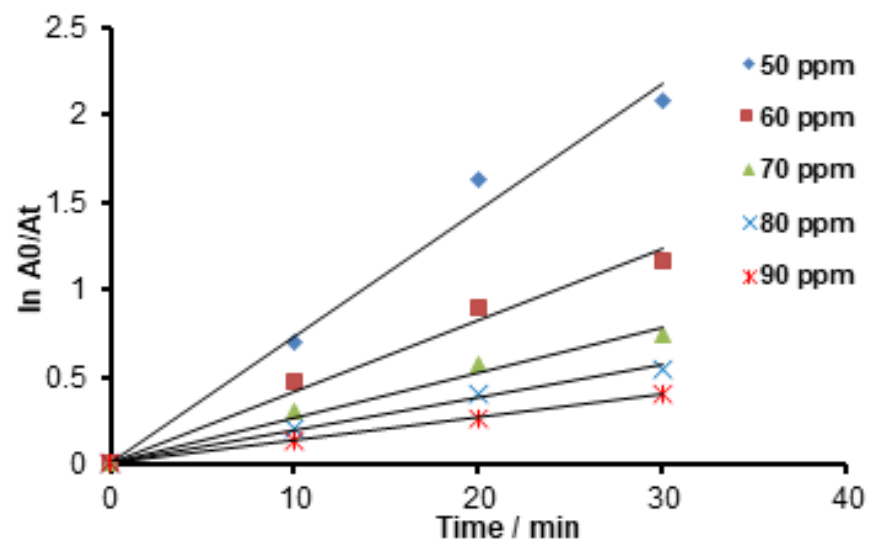

Fig 4. The change of $\ln \left(A_{0} / A_{t}\right)$ with irradiation time at a different concentration of brilliant cresyl blue by employment solar radiation, initial condition: $0.17 \mathrm{~g} / 100$ $\mathrm{cm}^{3}$ aluminum oxide and $\mathrm{pH}=10$

follows the pseudo-first order reaction according to Langmuir Hinshelwood relationship [14-15].

Fig. 5 shows the relationship between the initial concentration of brilliant cresyl blue dye at a various concentration with the percentage of photodegradation efficiency, the optimal value equal to $92.87 \%$.

\section{Effect of Initial pH on the Photocatalytic Degradation of Brilliant Cresyl Blue Dye}

Photocatalytic breakdown competence of brilliant cresyl blue was affected by $\mathrm{pH}$ changes. This alterations due to $\mathrm{pH}$ parameter could be cleared the relationship 


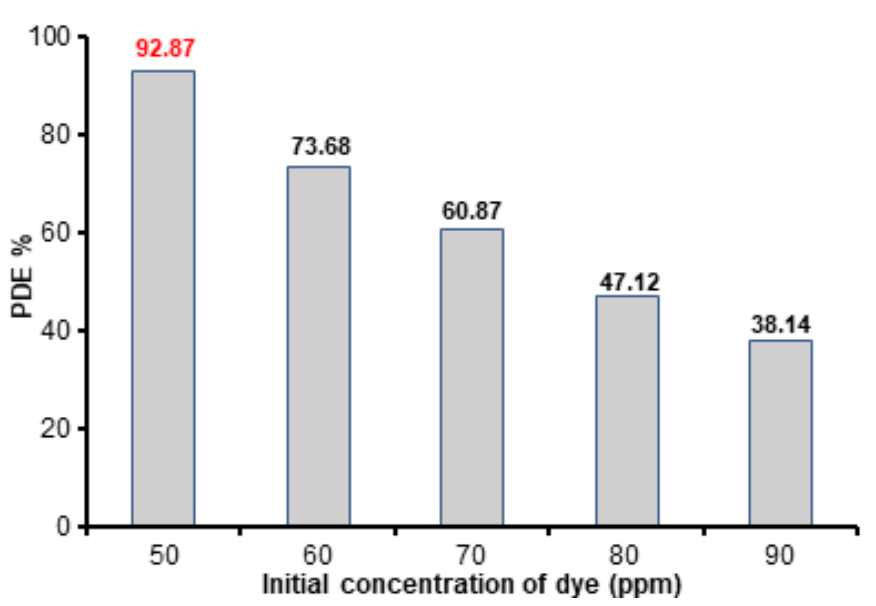

Fig 5. The change of Photocatalytic Degradation Efficiency with various concentration of brilliant cresyl blue

between the catalyst surface charge and the stain. The effect of initial $\mathrm{pH}$ on the photocatalytic degradation of the chosen brilliant cresyl blue dye has been analyzed by varying the initial $\mathrm{pH}$ range $4-11$. The effect $\mathrm{pH}$ on the rate of degradation of dyes has been shown in Fig. 6. Before, starting to explain the findings, it must determine the point of zero charges of $\mathrm{Al}_{2} \mathrm{O}_{3}$, from the literature is 8.6. The aluminum oxide surface has positively charged in acidic media $(\mathrm{pH}<8.5)$, whereas it has negatively charged under alkaline conditions $\mathrm{pH}>6.8$. The brilliant cresyl blue dye is cationic pigment. This type of stains has been recorded poor adsorption in the acidic environment. As a result, these reasons leads to a decrease in the photocatalytic degradation efficiency (PDE) of brilliant cresyl blue. At supreme $\mathrm{pH}$ value in the alkaline medium, more stain molecules would be adsorbed on to the catalyst surface which resulted in high photocatalytic degradation efficiency of dye. It was due to the electrostatic attraction of the negatively charged of $\mathrm{Al}_{2} \mathrm{O}_{3}$ with the cationic pigment.

The photocatalytic destruction of brilliant cresyl blue has been found to be maximal at $\mathrm{pH} 10$ in which photocatalytic competence equal to $91.67 \%$. This maximum of photocatalytic efficiency was caused by the produce the hydroxyl radical which is generated from oxidizing hydroxyl ions. On the other hand, increases absorption of brilliant cresyl blue tincture on the surface of aluminum oxide, the $\mathrm{pH}>=10$, in alkaline medium,

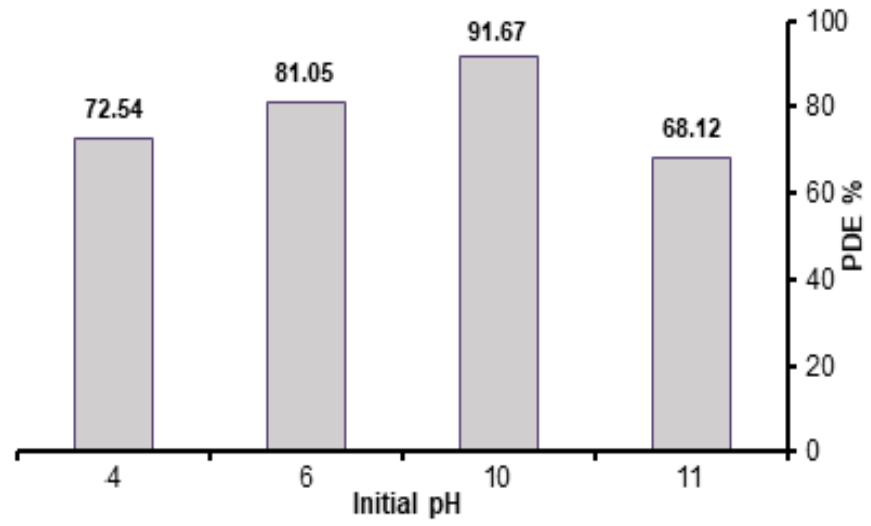

Fig 6. Photocatalytic degradation of brilliant cresyl blue dye at variation initial $\mathrm{pH}$ by usage solar radiation, initial Brilliant cresyl blue stain concentrations $=50 \mathrm{ppm}$, amount of photocatalyst aluminum oxide $=0.17 \mathrm{~g} / 100 \mathrm{~cm}^{3}$

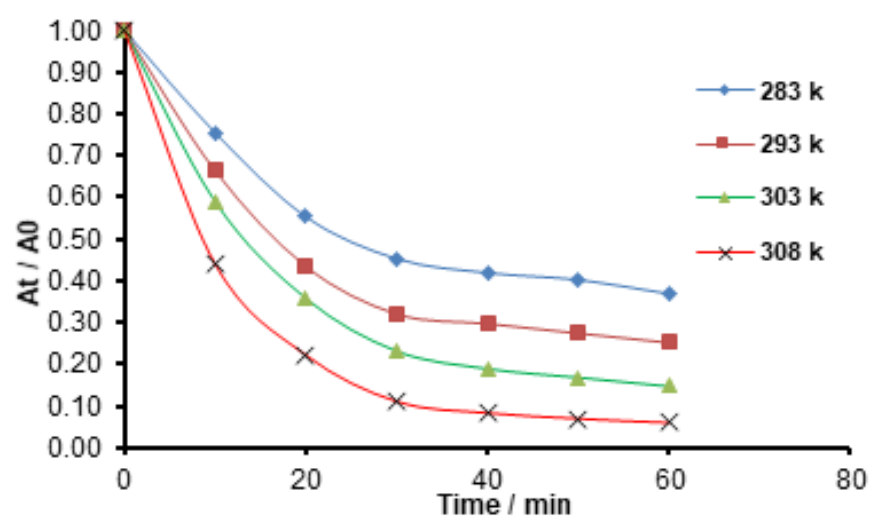

Fig 7. The change of $\left(A / A_{0}\right)$ with irradiation time at various temperature for photocatalytic destroy an average of bright cresyl blue by using solar energy, initial brilliant cresyl blue stain concentrations $=50 \mathrm{ppm}$, amount of photocatalyst aluminum oxide $=0.17 \mathrm{~g} / 100 \mathrm{~cm}^{3}$ and $\mathrm{PH}=10$

photocatalytic degradation of brilliant cresyl blue dye has been gradually decreasing due to the reduction of the brilliant cresyl blue stain that was absorbed on the negative charge surface aluminum oxide as well as at high $\mathrm{pH}$ values of hydroxyl radicals are so rapidly scavenged [16].

\section{Effect of Temperature on Photocatalytic Degradation of Brilliant Cresyl Blue}

A series of experiments were carried out to study the effect of temperature on the photocatalytic degradation rate of bright cresyl blue by using aluminum 
oxide suspension at a various temperature ranging from 283-308 K [17-19]. Fig. 7 has shown the impacting of temperature on the photocatalytic degradation rate of brilliant cresyl blue at a fixed initial concentration of 50 ppm and $0.17 \mathrm{~g} / 100 \mathrm{~cm}^{3}$ of aluminum oxide as a catalyst. Fig. 7 indicates that the photocatalytic breakdown average of flaming cresyl blue increases. This rise has accompanied with the rise in temperature due to a superfluity of generation of free radicals, and which lead to falling recombination process.

\section{- CONCLUSION}

The photocatalytic degradation process of brilliant cresyl blue pigment depends on the amount of catalyst mass and the optimum value equal to $0.17 \mathrm{~g} / 100 \mathrm{~cm}^{3}$ of aluminum oxide. Photocatalytic destruction of radiant cresyl blue stain obeyed the pseudo-first-order reaction. Photocatalytic degradation processes have decreased. This dropping is accompanied with increasing the concentration of Brilliant cresyl blue stain which due to the decrease of the concentration of $\mathrm{OH}^{-}$has adsorbed on the catalyst surface with $50 \mathrm{ppm}$ concentration of flaming cresyl blue pigment as an optimum value. The best account of $\mathrm{pH}$ of photocatalytic degradation equal to 10 . Photocatalytic degradation process of brilliant cresyl blue stain increases with the increase of light intensity because of the increase of photoelectron in the conduction band this lead to an increase of a number of electron-hole pairs, and a decrease of recombination process between photoelectron and a cavity in valance bundle. The best value of light intensity $8.44 \mathrm{~mW} / \mathrm{cm}^{2}$. The percentage competence of degradation reactive blue equals $92.87 \%$.

\section{- ACKNOWLEDGMENTS}

Sincerely and so thankful for the University of Babylon, College of Science for Women, Department of Chemistry for providing this opportunity the necessary infrastructural facilities such as laboratory work, library, and I.T. during my current study.

\section{- REFERENCES}

[1] Misriyani, Kunarti, E.S., and Yasuda, M., 2015, Synthesis of Mn(II)-Loaded TiXSi1- $\mathrm{XO}_{4}$ Composite acting as a visible light driven photocatalyst, Indones. J. Chem., 15 (1), 43-49.

[2] Gaya, U.I., and Abdullah, A.H., 2008, Heterogeneous photocatalytic degradation of organic contaminants over titanium dioxide: A review of fundamentals, progress and problems, $J$. Photochem. Photobiol., C, 9 (1), 1-12.

[3] Liao, W., Zeng, T., Wang, P., Tu, S., and Pan, W., 2010, Efficient microwave-assisted photocatalytic degradation of endocrine disruptor dimethyl phthalate over composite catalyst $\mathrm{ZrO} / \mathrm{ZnO}, J$. Environ. Sci., 22 (11), 1800-1806.

[4] Pereira, J.H.O.S., Vilar, V.J.P., Borges, M.T., González, O., Esplugas, S., and Boaventura, R.A.R., 2011, Photocatalytic degradation of oxytetracycline using $\mathrm{TiO}_{2}$ under natural and simulated solar radiation, Sol. Energy, 85 (11), 2732-2740.

[5] Yuliati, L., Roslan, N.A., Siah, W.R., and Lintang, H.O., 2017, Cobalt oxide-modified titanium dioxide nanoparticle photocatalyst for degradation of 2,4-dichlorophenoxyacetic acid, Indones. J. Chem., 17 (2), 284-290.

[6] Syafei, D., Sugiarti, S., Darmawan, N., and Khotib, M., 2017, Synthesis of $\mathrm{TiO}_{2} /$ carbon nanoparticle (C-dot) composites as active catalysts for photodegradation of persistent organic pollutant, Indones. J. Chem., 17 (1), 37-42.

[7] Mallakpour S., and Khadem, E., 2016, Carbon nanotube-metal oxide nanocomposites: Fabrication, properties and applications, Chem. Eng. J., 302, 344-367.

[8] Zhou W., Zhang L., Denghui J., Zhong, X., and Li, X., 2016, Enhanced photocatalytic degradation of organic dyes by palladium nanocrystals, J. Nanosci. Nanotechnol., 16 (7), 7497-7502.

[9] Chen, Y., Zhang, C., Huang, W., Yang, C., Huang, T., Situ, Y., and Huang, H., 2014, Synthesis of porous $\mathrm{ZnO} / \mathrm{TiO}_{2}$ thin films with superhydrophilicity and photocatalytic activity via a template-free sol-gel method, Surf. Coat. Technol., 258, 531-538.

[10] Ackacha, M.A., and Drmoon, M., 2012, Effect of important selected parameters on adsorption 
capacity of brilliant cresyl blue dye onto novel adsorbent: Tamarix aphylla leaves, IPCBEE, 48, 7175.

[11] Qin, L., Liu, M., Wu, Y., Xu, Z., Guo, X., and Zhang, G., 2016, Bioinspired hollow and hierarchically porous $\operatorname{MOx}(\mathrm{M}=\mathrm{Ti}, \mathrm{Si}) /$ carbon microellipsoids supported with $\mathrm{Fe}_{2} \mathrm{O}_{3}$ for heterogeneous photochemical oxidation, Appl. Catal., B, 194, 50-60.

[12] Rattan, V.K., Purai, A., Singh, H., and Manoochehri, M., 2008, Adsorption of dyes from aqueous solution by cow dung ash, Carbon Lett., 9 (1), 1-7.

[13] Al-Gubury, H.A., and Mohammed, Q.Y., 2016, Prepared coupled $\mathrm{ZnO}-\mathrm{Co}_{2} \mathrm{O}_{3}$ then study the photocatalytic activities using crystal violet dye, JCHPS, 9 (3), 1161-1165.

[14] Pei, C.C., and Leung, W.W.F., 2013, Photocatalytic degradation of Rhodamine $\mathrm{B}$ by $\mathrm{TiO}_{2} / \mathrm{ZnO}$ nanofibers under visible-light irradiation, Sep. Purif. Technol., 114, 108-116.

[15] Subramonian, W., Wu, T.Y., and Chai, S.P., 2017, Photocatalytic degradation of industrial pulp and paper mill effluent using synthesized magnetic $\mathrm{Fe}_{2} \mathrm{O}_{3}-\mathrm{TiO}_{2}$ : Treatment efficiency and characterizations of reused photocatalyst, $J$. Environ. Manage., 187, 298-310.

[16] Purnawan, C., Wahyuningsih, S., and Kusuma, P.P., 2016, Photocatalytic and photoelectrocatalytic degradation of methyl orange using graphite/ $\mathrm{PbTiO}_{3}$ composite, Indones. J. Chem., 16 (3), 347-352.

[17] Patil, P.N., Bote, S.D., and Gogate, P.R., 2014, Degradation of imidacloprid using combined advanced oxidation processes based on hydrodynamic cavitation, Ultrason. Sonochem., 21 (5), 1770-1777.

[18] Gosu, V., Gurjar, B.R., Surampalli, R.Y., and Zhang, T.C., 2014, $\mathrm{nFe}^{0} / \mathrm{GAC}$-mediated advanced catalytic per-oxidation for pharmaceutical wastewater treatment, J. Environ. Chem. Eng., 2 (4), 1996-2004.

[19] Al-Gubury, H.A., Study the activity of titanium dioxide nanoparticle using orange $G$ dye, 2016, MJS, 35 (2), 319-330. 